\title{
Eurostudia
}

\section{Grèce : le pluralisme religieux au défi de la crise migratoire}

\section{Nicolas Kazarian}

Volume 13, numéro 1-2, 2018-2019

Cultures en contact, entre régulations et représentations

URI : https://id.erudit.org/iderudit/1064490ar

DOI : https://doi.org/10.7202/1064490ar

Aller au sommaire du numéro

Éditeur(s)

Le Centre canadien d'études allemandes et européennes

ISSN

1718-8946 (numérique)

Découvrir la revue

Citer cet article

Kazarian, N. (2018). Grèce : le pluralisme religieux au défi de la crise migratoire. Eurostudia, 13(1-2), 93-114. https://doi.org/10.7202/1064490ar

\section{Résumé de l'article}

La Grèce revient régulièrement sur le devant de la scène médiatique. La crise économique et financière, ainsi que la crise migratoire qui touche ce pays du sud-est européen ont mis en lumière la place complexe du fait religieux, la gestion difficile du pluralisme des croyances, en ces terres majoritairement orthodoxes. Tiraillée entre repli identitaire et action humanitaire, la Grèce tente aujourd'hui de faire face à la question migratoire alors même que les pouvoirs publics ne cessent de revisiter le statut symbolique et légal de l'Église orthodoxe. Réforme de l'enseignement de la religion à l'école ou rémunération du clergé par l'État, la montée du pluralisme religieux en Grèce est perçue comme une dimension de la sécularisation promue dans le contexte européen. Cependant, la crise migratoire constitue-t-elle un danger de plus en faveur du renforcement du pluralisme religieux ou un défi pour l'Église orthodoxe de dépasser les crispations identitaires au travers de son engagement humanitaire?
Tous droits réservés $@$ Le Centre canadien d'études allemandes et européennes, 2019
Ce document est protégé par la loi sur le droit d'auteur. L'utilisation des services d’Érudit (y compris la reproduction) est assujettie à sa politique d'utilisation que vous pouvez consulter en ligne.

https://apropos.erudit.org/fr/usagers/politique-dutilisation/ 


\section{Nicolas Kazarian}

Institut de Relations Internationales et Stratégiques

\section{Résumé}

La Grèce revient régulièrement sur le devant de la scène médiatique. La crise économique et financière, ainsi que la crise migratoire qui touche ce pays du sud-est européen ont mis en lumière la place complexe du fait religieux, la gestion difficile du pluralisme des croyances, en ces terres majoritairement orthodoxes. Tiraillée entre repli identitaire et action humanitaire, la Grèce tente aujourd'hui de faire face à la question migratoire alors même que les pouvoirs publics ne cessent de revisiter le statut symbolique et légal de l'Église orthodoxe. Réforme de l'enseignement de la religion à l'école ou rémunération du clergé par l'État, la montée du pluralisme religieux en Grèce est perçue comme une dimension de la sécularisation promue dans le contexte européen. Cependant, la crise migratoire constitue-t-elle un danger de plus en faveur du renforcement du pluralisme religieux ou un défi pour l'Église orthodoxe de dépasser les crispations identitaires au travers de son engagement humanitaire?

\section{Abstract}

Greece comes back regularly to the front page. The economic and financial crisis, as well as the migration crisis affecting this South-Eastern European country have highlighted the complex place of religion, the difficult management of religious pluralism in this predominantly Orthodox country. Torn between identity isolation and humanitarian action, Greece today is trying to cope with the migration issue while the state authorities keep revisiting the symbolic and legal status of the Orthodox Church. Whether through the reform of religious education in public schools or the 
remuneration of the clergy by the state, the rise of religious pluralism in Greece is considered as a dimension of secularization promoted in and by the European sphere. However, is the migration crisis strengthening religious pluralism or challenging the Orthodox Church to overcome identity tensions through its humanitarian commitment?

\section{Introduction}

Le 16 avril 2016, trois hiérarques chrétiens, le Pape François, le Patriarche œcuménique Bartholomée, l'Archevêque Jérôme d'Athènes désiraient attirer l'attention de l'opinion internationale sur la crise migratoire qui touche encore aujourd'hui la Grèce, en se retrouvant sur l'île de Lesbos. Dans la déclaration commune publiée à l'issue de leur rencontre, nous pouvons lire :

L'Europe affronte aujourd'hui l'une de ses plus sérieuses crises humanitaires depuis la fin de la Seconde Guerre mondiale. Pour répondre à ce grave défi, nous appelons tous les disciples du Christ à se souvenir des paroles du Seigneur, sur lesquelles nous serons jugés un jour : 'Car, j'avais faim, et vous m'avez donné à manger; j'avais soif, et vous m'avez donné à boire; j'étais un étranger, et vous m'avez accueilli; j'étais nu, et vous m'avez habillé; j'étais malade, et vous m'avez visité; j'étais en prison, et vous êtes venus jusqu'à moi... Amen, je vous le dis : chaque fois que vous l'avez fait à l'un de ces plus petits de mes frères, c'est à moi que vous l'avez fait' (Matthieu 25, 35-36.40)1.

Ce passage attaque implicitement l'accord turco-européen qui avait été signé un mois plus tôt, le 18 mars 2016. Tous les nouveaux migrants irréguliers ne demandant pas l'asile ou dont la demande d'asile a été jugée infondée ou

\footnotetext{
1 «Déclaration commune de Sa Sainteté Bartholomée, Patriarche œcuménique de Constantinople, de Sa Béatitude Hyeronimos, Archevêque d'Athènes et de toute la Grèce et du Saint Père François ", Lesvos, 16 avril 2016; $\quad$ http://w2.vatican.va/content/francesco/fr/speeches/2016/april/documents/papafrancesco 20160416 lesvos-dichiarazione-congiunta.html
} 
irrecevable et étant passés par la Turquie vers les îles grecques depuis le 20 mars 2016 devaient être renvoyés vers la Turquie.

La déclaration commune fait aussi référence à la crise financière qui touche encore aujourd'hui la Grèce, et qui a entraîné une forte polarisation de la population et de l'échiquier politique. Même si la Grèce n'est qu'un pays de transit pour les migrants, leur endiguement en raison de la fermeture des frontières au nord entraîne un effet d'accumulation qui pèse mécaniquement sur la composition, la perception et la gestion du pluralisme religieux dans le pays (Clogg 2002).

La question du pluralisme religieux s'est imposée comme le reflet d'un contexte local en continuelle mutation. La diversité historique des communautés religieuses se voit bouleversée par des phénomènes à la fois internes, comme le niveau de pratique religieuse, et externes, comme un accroissement de la population religieuse par voie de migration. Dans certains pays, comme la France, la question du pluralisme religieux ne porte pas tant sur la position hégémonique de l'Église catholique, que sur l'intégration des membres de la communauté musulmane. En Grèce, le rapport de force n'est certainement pas que démographique, il est aussi symbolique, voire géopolitique, notamment dans le cadre de la place de l'Islam dans le pays comme levier de relation avec son grand voisin, la Turquie. Mais au-delà des considérations théologiques qui prévalent dans l'articulation des relations entre les religions comme des ensembles de vérité séparés, voire concurrentiels, c'est avant tout sur le champ politique, législatif et de l'identité nationale que se construisent les évolutions du pluralisme religieux. Ce dernier est perçu comme une chance ou comme un danger, il n'en demeure pas moins un principe de (co-)existence plus ou moins pacifique à l'intérieur duquel se construit une expérience cultuelle plus ou moins libre. La liberté religieuse devient alors le corolaire du pluralisme religieux (Banchoff 2008). 
Tiraillée entre repli identitaire et action humanitaire, la Grèce tente de faire face à la question migratoire alors même que les pouvoirs publics ne cessent de revisiter le statut symbolique et légal de l'Église orthodoxe. Réforme de l'enseignement de la religion à l'école, rémunération du clergé par l'État, séparation Eglise-Etat, la montée du pluralisme religieux en Grèce est perçue comme une dimension de la sécularisation promue dans le contexte européen. Cependant, la crise migratoire constitue-t-elle un danger de plus renforçant le pluralisme religieux ou un défi pour l'Église orthodoxe de dépasser les crispations identitaires?

\section{Un paysage religieux dominé par l'orthodoxie}

En Grèce, l'appartenance religieuse constitue un marqueur essentiel de l'identité ethnique. Cette relation entre religion et ethnie s'explique notamment par l'héritage de la période ottomane marquée par l'imposition du système communautaire du millet (Ursinus 1993 : 61-64). Au XIXe puis au XXe siècle, les tensions issues du nationalisme ont achevé de consolider la synthèse entre identité nationale et orthodoxie dans la Grèce moderne (Roudometoff $2010: 27$ ).

La composition ethnoreligieuse de la Grèce est particulièrement homogène avec une large majorité de la population grecque orthodoxe (Kazarian 2018). Les autorités grecques n'ont pas réalisé de recensement linguistique et religieux depuis 1951. Selon le Pew Research Center, 87\% des Grecs sont chrétiens orthodoxes, soit une population d'environ 9880000 fidèles rattachés pour la majeure partie d'entre eux à l'Église autocéphale orthodoxe de Grèce ${ }^{2}$. Le groupe chrétien orthodoxe compte également un courant de traditionalistes appelés « anciens calendaristes », qui représentent entre 700000 et 1 million de fidèles. Elle est officiellement séparée

2 Cf. Pew Research Center, "Orthodox Christianity in the 21 $1^{\text {st }}$ Century », 8 novembre 2017, p. 23; https://www.pewforum.org/2017/11/08/orthodox-christianity-in-the-21st-century/ 
de l'Église de Grèce (Ware 2002). Parmi les autres familles chrétiennes, il y aurait 50000 Grecs catholiques romains, 35000 Arméniens apostoliques et 20000 protestants. On estime aussi le nombre de Témoins de Jéhovah à 70000 fidèles, ainsi que le nombre de Juifs à 5000 (Yannas 2010 : 113).

La composante musulmane de la population grecque jouit d'un statut particulier puisque l'État grec reconnaît officiellement la « minorité » musulmane de Thrace occidentale, comme le prévoit le Traité de Lausanne de 1923 (Aknögül 1999). Ce groupe compterait aujourd'hui de 100000 à 125000 personnes, réparties entre trois départements jouxtant la Turquie et la Bulgarie : Xanthi (entre 40000 et 45000 musulmans), Rhodope (60000 et 65000 musulmans) et Évros (8000 et 16000 musulmans). Cette «minorité » se subdivise en trois groupes ethnolinguistiques: les turcophones, majoritairement dans le département de Rhodope (35\% de la minorité), les Pomaks centrés dans le département de Xanthi, parlant un dialecte bulgare ( $37 \%$ de la minorité), et les Tsiganes, parlant le romani (25\% de la minorité) (Anagnostou et Triandafyllidou $2006: 2)^{3}$.

De facto, il est difficile d'avoir un tableau clair de la diversité minoritaire en Grèce, tant culturellement que religieusement. Les organisations internationales proposent différentes catégories pour tenter de décrire le paysage communautaire du pays. Elles sont rapidement confrontées à la multiplication de ces critères (religieux, linguistiques, territoriaux, etc.), ainsi qu'à l'absence de revendications de certaines communautés. Il y a des pontiques en Grèce, mais pour des raisons de politique intérieure et d'identité nationale, ils ne sont pas dissociés de la population majoritaire. D'ailleurs, ils ne sont jamais traités comme une communauté à part. D'autre part, pour la définition des Grecs pontiques : Grecs originaires du nord de

\footnotetext{
${ }^{3}$ Il convient de noter que ces estimations datent. Pour des raisons propres à la lecture du phénomène minoritaire par l'Etat grec, une caractérisation plus spécifique des communautés à l'intérieur de la minorité musulmane n'est pas envisageable. D'autre part, la majorité des Tsiganes grecs sont chrétiens orthodoxes, seule une partie d'entre eux sont musulmans. Ils sont d'ailleurs concentrés en Thrace.
} 
la Turquie et des rives de la mer Noire. Ils sont aussi appelés « Russo-Pontiques » à cause de leur présence dans l'ancien bloc soviétique (Bruneau 1998).

La Constitution de la Grèce date de 1975. Elle reconnaît officiellement une place dominante à la religion orthodoxe. Outre une invocation à la « Trinité Sainte, Consubstantielle, et Indivisible » placée en ouverture de la Constitution, l'article 3 définit les rapports entre l'État et l'Église. Elle reconnait notamment que la religion «dominante » en Grèce est l'Orthodoxie, représentée par l’Église orthodoxe de Grèce. Le texte va plus loin en définissant son administration: "Elle est autocéphale et administrée par le Saint-Synode, qui est composé des Évêques en fonction, et par le Saint-Synode permanent [...] comme il est prescrit par la Charte statutaire de l'Église »(art.3,1). Dès lors, l'Église orthodoxe est considérée comme une personne morale de droit public comme le précise la loi 590/1977 relative à la Charte statutaire de l'Église orthodoxe de Grèce.

Ce statut de personne morale de droit public est aussi accordé à l'Islam (en Thrace occidentale) et au Judaïsme. Cependant, les autres groupes religieux sont considérés comme des personnes morales de droit privé. Au contraire, l'Église orthodoxe de Grèce possède des privilèges découlant de son statut : le paiement des salaires du clergé par l'État ou certaines exemptions fiscales, par exemple, qui ne sont propres qu'à elle. Ce statut renforce sa position dominante par rapport aux autres religions et à la société grecque dans son ensemble. Pour autant, un accord signé entre l'Église orthodoxe de Grèce et le gouvernement d'Alexis Tsipras en novembre 2018 pourrait faire évoluer la position préférentielle de l'orthodoxie, marquant la progressive séparation de l'Eglise et de l'Etat défendue en particulier par le parti du Premier ministre, Syriza. Au titre de cet accord, les prêtres pourraient ne plus être rémunérés par l'État au motif de la neutralité de l'autorité publique. 
L'article 5 de la Constitution aborde la question des libertés publiques et reconnaît le droit à la liberté de convictions religieuses. L'article 13 revient sur la liberté de religion et de conscience et fait la distinction entre les religions «connues » et les autres : «Toute religion connue est libre, et les pratiques de son culte s'exercent sans entrave sous la protection des lois. Il n'est pas permis que l'exercice du culte porte atteinte à l'ordre public et aux bonnes mœurs. Le prosélytisme est interdit » $(\operatorname{art.13}, 2)$. Le texte exclut aussi toute forme d'offense «à la religion chrétienne et à toute autre religion connue»(art.14,3a). Le texte ne donne pas une définition claire des religions «connues » et il n'existe pas de mécanisme d'acquisition de ce statut qui soit reconnu indirectement lorsque le ministre de l'Éducation et des Cultes accorde des autorisations d'acquisition de « lieux de culte». Les catholiques romains, les pentecôtistes, les méthodistes, les évangéliques et les témoins de Jéhovah ont ainsi été reconnus comme « religions connues». Jusqu'en 2006, les autorités orthodoxes pouvaient influer sur la construction des lieux de cultes d’autres religions (art.27, loi n³467/2006).

Dans le domaine de l'enseignement, la combinaison des articles 3 et 16 de la Constitution instaure une certaine domination de l'Église orthodoxe de Grèce. En effet, elle lie, d'une part, la position de religion dominante de l'Église orthodoxe de Grèce, avec l'élaboration de la conscience nationale et religieuse des élèves. L'Église orthodoxe de Grèce a aussi une influence normative dans le domaine de la recherche universitaire et de l'enseignement supérieur, notamment sur les universités d'État. La loi n`3432/2006 réévalue et organise l'enseignement ecclésiastique. Le contenu de l'instruction religieuse dans le primaire et le secondaire est dominé par la religion orthodoxe. D'ailleurs, le respect des normes orthodoxes doit être suivi par l'enseignement religieux des élèves, les ouvrant à une conscience orthodoxe relevant de la doctrine de l'Église orthodoxe de Grèce. Les cours $d$ 'instruction religieuse ne sont pas obligatoires pour les élèves appartenant à des religions minoritaires (loi n¹566/1985). 
D'autre part, le service militaire et la crémation sont deux thèmes liés à la protection de la liberté religieuse. La loi n`2510/1997 permet, en effet, aux objecteurs de conscience pour des raisons religieuses d'accomplir le service militaire sans arme, ou de favoriser le service civique. Cette loi a notamment été promulguée suite aux revendications des Témoins de Jéhovah. S'agissant du droit à la crémation, cette dernière pratique s'oppose au rite traditionnel d'enterrement, tel que reconnu par l'orthodoxie. Depuis 2006, néanmoins, il est possible aux personnes l'ayant déclaré préalablement d'opter pour un enterrement nonreligieux. De même, la pratique de la crémation, contre laquelle l'Église orthodoxe se levait pour des raisons dogmatiques, a été rendue possible (loi n³448/2006, art.35).

Le mariage civil est reconnu comme valide depuis 1982, au même titre que les cérémonies religieuses (loi ${ }^{\circ} 1250 / 1982$ ). Le mariage religieux ne nécessite pas de mariage civil préalable. L'année suivante, le gouvernement socialiste fait voter une loi réglementant les procédures de divorce et visant à établir l'égalité des époux (loi n¹329/1983).

\section{Le pluralisme religieux en débat en Grèce}

Le pluralisme religieux fait de plus en plus débat en Grèce, car il est utilisé par le gouvernement d'Alexis Tsipras comme un moyen pour imposer la neutralité religieuse de l'État grec. De nombreux débats cristallisent le rapprochement entre pluralisme religieux et sécularisation. Nous ne retiendrons ici que trois aspects : la question du prosélytisme, celle de l'enseignement de la religion à l'école et enfin la place et le statut de la communauté musulmane en Grèce.

Dans ce contexte, la question du prosélytisme est de première importance, en tant que l'expression d'une hétérogénéité religieuse dans sa relation frontale avec la religion dominante. L'article 13,2 de la Constitution interdit le prosélytisme, 
sans pour autant en donner une définition concrète, permettant ainsi la confusion entre la liberté religieuse des communautés religieuses minoritaires et l'action prosélyte. La frontière entre les deux est donc ténue et elle est en particulier interrogée par des membres de communautés non-orthodoxes. Ainsi, la volonté des minorités religieuses de propager leur foi peut être considérée comme une forme de prosélytisme dès lors qu'elle entre en compétition avec la religion majoritaire (Necessity Acts $\mathrm{n}^{\circ} 1363 / 1938$ et $\left.\mathrm{n}^{\circ} 1672 / 1939\right)$. La pénalisation du prosélytisme s'inscrit, par conséquent, comme une forme de protection de l'Orthodoxie non seulement en tant que religion majoritaire, mais aussi comme symbole identitaire.

La CEDH s'est vivement opposée à une telle pratique. Dans l'affaire «Kokkinakis c. la Grèce », Minos Kokkinakis, membre de la communauté des Témoins de Jéhovah, porte plainte contre l'État grec de sa condamnation pour prosélytisme. Bien que le Conseil d'État grec la considère comme une «religion connue » (arrêts n²105, 2106/1975 et 3601/1990), les arrestations et les condamnations de ses membres pour prosélytisme sont nombreuses et répondent à une jurisprudence relative à l'action prosélyte en particulier des Témoins de Jéhovah en opposition à l'orthodoxie ${ }^{4}$. L'arrêt de la CEDH reconnaît, entre autres, qu'il y a bien eu violation de l'article 9 de la Convention européenne des Droits de l'homme (Convention européenne) dans cette affaire : «Dès lors, il n'a pas été démontré que la condamnation de l'intéressé se justifiait, dans les circonstances de la cause, par un besoin social impérieux. La mesure incriminée n'apparaît donc pas proportionnée au but légitime poursuivi, ni, partant, "nécessaire, dans une société démocratique", "à la protection des droits et libertés d'autrui" » ${ }^{5}$.

\footnotetext{
${ }^{4}$ Voir l'analyse de cette jurisprudence par la CEDH : « Kokkinokis c. la Grèce », CEDH, Conseil de l'Europe, 25 mai 1993 (Requête $n^{\circ} 14307 / 88$ ), § 17-21.

${ }^{5}$ « Kokkinokis c. la Grèce », CEDH, Conseil de l’Europe, 25 mai 1993 (Requête n¹4307/88), § 49.
} 
La Constitution grecque ne prévoit pas explicitement un enseignement orthodoxe, mais celui-ci existe sur la base de la combinaison de l'article 3 (religion orthodoxe comme religion dominante de la population grecque) avec l'article 16 (développement de la conscience religieuse des élèves). La loi n¹566/85 de 1985 exige le respect de la norme orthodoxe dans l'enseignement religieux et la formation des élèves à une conscience spécifiquement orthodoxe en accord avec la doctrine de l'Église.

Les relations entre le gouvernement d'Alexis Tsipras et l'Eglise de Grèce se sont détériorées ces dernières années en raison, notamment, du plan de transformation des cours de religion donnée dans le primaire et le secondaire. Le traitement $\mathrm{du}$ pluralisme religieux s'apparente alors plus que jamais à «un processus historique de dé-monopolisation » (Berger et Luckmann 1967 : 117). Il s'agit en effet de transformer ce qui relevait avant tout des cours de catéchisme en cours d'introduction à différents cadres culturels, systèmes de valeurs et doctrine des principales religions $\mathrm{du}$ monde. Il s'agissait $\mathrm{d}^{\prime}$ abolir la dimension confessionnelle de l'éducation religieuse, d'en diminuer les heures d'enseignement et de rendre les cours de religions facultatifs. Ce projet gouvernemental a entraîné des réactions extrêmement vives de la part de l'Eglise de Grèce, mettant en danger le modus vivendi entre Église et État. Il est intéressant de remarquer que l'archevêque Jérôme a récemment entamé un rapprochement avec certains mouvements radicaux de droite, délaissant la position plus modérée qui le caractérisait jusque-là, son discours sur ces questions reprenant les tendances (ultra-)conservatrices présentes dans le Saint-Synode de l'Église de Grèce. Pour faciliter ses négociations avec l'Eglise de Grèce, le gouvernement d'Alexis Tsipras a remercié son ministre de l'Education Nikos Philis en novembre 2016 que l'archevêque Jérôme accusait d'anticléricalisme. Le parti d'extrême gauche Syriza a alors retiré les éléments les plus sécularisés de son programme au nom de la protection de la cohésion sociale dans un moment critique pour l'économie du 
pays. La coalition politique portée par Syriza était elle aussi fragilisée en raison de ces problématiques, même si son long travail de sécularisation de la société grecque semble porter ses fruits sur d'autres chantiers comme celui du salariat $d u$ clergé par l'Etat grec ${ }^{6}$. En revanche, le Conseil d'Etat a fini par annuler définitivement la réforme éducative portée par le gouvernement de Tsipras en 2018. Pour Evanthi Tsaliki, même si l'éducation religieuse en Grèce paraît conforme aux directives européennes, il semblerait que la référence aux autres religions reste minime tant l'orthodoxie est une composante centrale de l'identité nationale grecque. Selon cette dernière :

Il est évident que le sujet de l'éducation religieuse dans les écoles ordinaires grecques ne s'écarte pas de son orientation chrétienne orthodoxe. Elle contient quelques références à d'autres religions et croyances ainsi qu'à certains problèmes sociaux qui sont à nouveau abordés dans leur dimension chrétienne orthodoxe (Tsaliki 2017 : 50).

L'existence d'une minorité musulmane de Thrace occidentale résulte du Traité de Lausanne du 30 janvier 1923 qui exclut expressément de l'échange obligatoire de populations entre la Grèce et la Turquie prévu par le traité la population musulmane vivant en Thrace occidentale. Ce groupe comptait 86000 personnes en 1923 et en compterait, aujourd'hui de 100000 à 125 000. En Grèce, le traitement de cette minorité découle encore aujourd'hui des termes de la troisième section du Traité de Lausanne, établissant une série de droits positifs s'agissant de la pratique religieuse et du droit à l'éducation dans la langue de la communauté minoritaire. Plus particulièrement, ce droit s'étend à la juridiction civile et religieuse des muftis et à un système d'enseignement mixte géré conjointement par les représentants de l'État grec et par ceux de la minorité (Meinardus 2002 : 82-83).

\footnotetext{
${ }^{6}$ Makrides, V. (2018), «L'orthodoxie grecque en transition ? Le gouvernement Syriza et les relations entre l'Eglise et l'Etat. », SciencesPo - L'Enjeu mondial (en ligne); https://www.sciencespo.fr/enjeumondial/en/odr/l-orthodoxie-grecque-en-transition-le-gouvernementsyriza-et-les-relations-entre-l-eglise-et-l-e
} 
On compte en Thrace 3 muftis, 270 imams et environ 300 mosquées. Il y a également 215 écoles minoritaires d'enseignement élémentaire, deux collèges, deux lycées et deux séminaires.

Bien que les membres de la minorité musulmane soient pleinement citoyens grecs, la Turquie est autorisée, par le Traité de Lausanne à avoir un consulat général à Komotini et à s'impliquer sur des sujets concernant la communauté musulmane. Cette présence n'a pas empêché une crispation des gouvernements grecs successifs par rapport au traitement de la minorité musulmane dans le sillage de la Question chypriote et des relations gréco-turques (Bertrand 2003 : 123-129; Kazarian 2012). Corrélativement, le sort de la minorité musulmane découle aussi du traitement de la communauté orthodoxe grecque de Turquie.

L'État grec est confronté non seulement à la reconnaissance minoritaire de la communauté musulmane, mais surtout à ses transformations identitaires internes, survenues dans les années 1950. La communauté musulmane revendique progressivement une identité proprement turque. Les autorités grecques soutiennent quant à elles le caractère musulman de la minorité sur la base du Traité de Lausanne. C'est pourquoi la Grèce interdit l'utilisation des mots «turc » et «Turquie » dans les noms des associations formées par la minorité, dans la mesure où la dimension turque de la communauté musulmane n'en représente qu'une composante. L'utilisation de ces termes constitue selon les tribunaux grecs une menace d'atteinte à l'ordre public et à l'intégrité territoriale ${ }^{7}$. En réponse, les membres de la communauté musulmane en appellent à la CEDH qui a condamné la Grèce à plusieurs reprises ${ }^{8}$. C'est notamment le cas dans l'affaire opposant

\footnotetext{
${ }^{7}$ Cf. la décision 36/1986 prise en 1986 par la Cour d'Appel de Thrace.

${ }^{8}$ La Cour a reconnu le droit à la minorité de s'appeler « turque » : « Sadik Ahmet v. Greece », CEDH, Conseil de l'Europe,15 novembre 1996 (Requête n¹8897/91) (J'ai remplacé par le numéro de la requête. À quel paragraphe faites-vous référence? Cette décision de la cour ne visant pas explicitement le droit de s'appeler " turc »); "Serif contre Grèce », CEDH, Conseil de l'Europe, 14 mars 2000 (Requête n³8178/97); et « Agga v. Greece », CEDH, Conseil de l’Europe, 13 octobre 2006 (Requête n³3331/02)
} 
Ahmet Sadik à la Grèce. Ce dernier, s'étant présenté d'abord comme candidat indépendant, puis comme candidat « turc » aux élections législatives de juin 1989 et de juillet 1990, il se voulait une figure indépendante à la tête du «Parti de l'Amitié et de l’Égalité ». Mort accidentellement en 1995, il internationalisa la cause de la minorité musulmane en Grèce par le biais d'une plainte déposée auprès de la CEDH. La Cour a reconnu le droit à la minorité de s'appeler « turque » et donc le droit à l'identification ethnique, en accord avec l'article 11 de la Convention européenne ${ }^{9}$. Une stratégie identitaire est alors mise en œuvre par les membres turcs de la communauté musulmane. Ainsi, de nombreux témoignages, relevés notamment par l'experte indépendante sur les minorités Gay McDougall, font état de pressions exercées sur les Pomaks et les Tsiganes pour apprendre et parler le turc et se définir comme turcs, influant sur la transformation du pluralisme à un niveau intracommunautaire ${ }^{10}$.

Dans le prolongement des questions liées à l'identification ethnique, la nomination des muftis des villes de Xanthi, Komotini et Didimochito constitue une nouvelle fragmentation de la minorité musulmane (loi n²345/1920, remplacée par la loi $\left.n^{\circ} 1920 / 1991\right)$. Faisant partie du corps administratif et possédant le statut de fonctionnaire, ils sont nommés pour une durée de dix ans par le Ministère de l'Éducation et des Affaires religieuses, en accord avec les dirigeants de la communauté musulmane. Cependant, la Turquie revendique le déroulement d'une procédure de vote pour l'élection du mufti. Une partie de la minorité a élu

\footnotetext{
9 « Sadik Ahmet v. Greece », CEDH, Conseil de l’Europe, 15 novembre 1996 (Requête n 18897/91). Voir aussi "Emin et autres c. Grèce », CEDH, Conseil de l'Europe, 27 mars 2008 (Requête n`34144/05) et " Association turque de Xanthi c. Grèce », CEDH, Conseil de l'Europe, 27 mars 2008 (Requête n² 26698/05).

${ }^{10}$ Gay McDougall, « Promotion et protection de tous les Droits de l'homme, civils, politiques, économiques, sociaux et culturels y compris le droit au développement. Mission en Grèce », Conseil des Droits de l'Homme, ONU, publié le 18 février 2009 [A/HRC/10/11/Add.3], $§ \quad 15$; https://undocs.org/A/HRC/10/11/Add.3
} 
ce que les autorités de Grèce nomment des "pseudo-muftis »" ${ }^{11}$ Néanmoins, le principal problème de la communauté musulmane touche désormais l'application de la charia, notamment dans son application aux femmes musulmanes ${ }^{12}$. Comme le font remarquer de nombreux rapports sur cette question, il est fortement recommandé de retirer la compétence judiciaire aux muftis dans la mesure où la loi islamique semble incompatible avec la Constitution grecque, avec le droit et les standards internationaux, affaiblissant la légitimité du pluralisme en Grèce ${ }^{13}$.

Les membres de la communauté se sentent discriminés. Pour les sortir de leur isolement, les autorités grecques entendent revaloriser l'enseignement à l'intérieur de la minorité, notamment sur le plan linguistique, grâce à un enseignement bilingue. La maîtrise de la langue grecque permet d'ailleurs à ses membres une meilleure insertion dans la société et sur le marché du travail.

\section{Migration et pluralisme religieux}

Anciennement pays d'émigration, la Grèce est devenue, depuis une vingtaine d'années, une terre d'immigration, du fait de sa position géographique et du pouvoir d'attraction européen. Plus d'un million d'immigrés vivent en Grèce, soit près de $10 \%$ de la population. Près de 200000 d'entre eux ne sont pas régularisés tandis que le nombre de sans-papiers n'a cessé d'augmenter ces

\footnotetext{
${ }^{11}$ Voir les décisions de la CEDH s'agissant des condamnations de la Grèce dans ces affaires : « Serif contre Grèce ", CEDH, Conseil de l’Europe, 14 mars 2000 (Requête n³8178/97); et "Agga v. Greece », CEDH, Conseil de l'Europe, 13 octobre 2006 (Requête n`33331/02)

${ }^{12}$ Réminiscence de la période ottomane, le droit islamique est aujourd'hui en vigueur en Thrace en tant que système judiciaire communautaire (loi $\mathrm{n}^{\circ} 2345 / 1920$ ) assuré par le mufti pouvant arbitrer les affaires liées au droit de la famille.

${ }^{13}$ Gay McDougall, « Promotion et protection de tous les Droits de l'homme, civils, politiques, économiques, sociaux et culturels y compris le droit au développement. Mission en Grèce ", Conseil des Droits de l'Homme, ONU, 18 février 2009 [A/HRC/10/11/Add.3], § 74; Thomas Hammarberg, « Report following his visit to Greece », Commissioner for Human Rights, Council of Europe, 19 février 2009 [CommDH(2009)9], §28-36; https://rm.coe.int/16806db821
} 
dernières années avec de nombreuses arrivées, provenant surtout du Pakistan, d'Afghanistan et d'Irak, du moins avant la crise syrienne débutée en 2011.

Il y aurait ainsi de 700000 à 800000 immigrés réguliers en Grèce, en incluant les 150000 immigrés issus des dernières régularisations massives de 2005 et 2006 (loi n`3386/2005 et loi n`3536/2007). Selon les dernières données de l’Institut de la Politique d'immigration, 60\% des immigrés en Grèce sont des Albanais (83\% des immigrés viennent de l'Albanie et de l'Europe de l'Est). Les motifs principaux de l'immigration sont le travail salarié (70\%), l'unification familiale et le travail indépendant (12\%).

Il convient également de mentionner cette particularité de l'immigration grecque, les «étrangers d'origine grecque », albanais, bulgares, personnes issues des pays de l'est et de la région de la mer noire (les Grecs pontiques) pour qui il existe une procédure de régularisation spécifique (Bruneau 1998). Néanmoins, les difficultés relatives à l'obtention des permis de séjour de longue durée posent encore problème. En effet, entre 1991 et 2006, seuls 400 immigrés ont réussi à obtenir un tel permis. En outre, les immigrés sont marginalisés dans leurs procédures face à l'État et sont confrontés à des difficultés tant sur le plan du marché du travail, que dans le domaine de l'enseignement ${ }^{14}$.

Néanmoins, ce qui est le plus préoccupant sur le plan des mutations du pluralisme concerne la situation des « immigrés de seconde génération » (200 000 enfants d'immigrés vivent en Grèce et sont nés dans le pays). En effet, la Grèce est un pays où s'applique strictement le droit du sang : le fait de naître en Grèce ne suffit pas pour l'acquisition de la nationalité. Les «immigrés de la seconde génération », même nés et élevés en Grèce, sont donc soumis aux mêmes règles que les immigrés de la première génération (Décret présidentiel n¹31/2005). Ils

\footnotetext{
${ }^{14}$ Quatrième rapport sur la Grèce, ECRI, Conseil de l’Europe, 15 septembre 2009 [CRI(2009)31], §39-50.
} 
semblent avoir du mal à se voir transmis les certificats de naissance, voire d'acquérir des permis de séjour de longue durée ${ }^{15}$. La procédure de naturalisation n'est pas plus facile.

Deux ans après le début de la guerre en Syrie en 2011, le nombre d'arrivées sur les rivages grecs avait déjà considérablement augmenté. Les dirigeants en Europe et en Grèce manquent encore d'un plan d'action concret pour gérer les flux de personnes fuyant la guerre. On estime que 850000 réfugiés et migrants sont passés par la mer en 2015 et plus de 168000 en octobre 2016. En moyenne, plus de 400 personnes arrivent encore de manière hebdomadaire en Grèce et 20\% d'entre elles sont des enfants. Mais la Grèce n'est qu'un pays de transit avant une relocalisation des migrants vers d'autres pays européens. Pour autant, l'engagement européen de septembre 2015 qui visait à relocaliser 64000 migrants de Grèce vers d'autres pays de l'UE ne s'est tout simplement pas réalisé. En novembre 2016, seules 5719 personnes avaient pu bénéficier de ce plan. Les migrants sont donc dans l'obligation soit de poursuivre leur chemin à leurs risques et périls, soit de s'installer à court ou moyen terme en Grèce. Il est vrai que l'accord turco-européen de mars 2016 a considérablement diminué la densité du flux migratoire, mais les délais de traitement des demandes d'asile sont longs et entraînent nombre de frustrations tant chez les Grecs que chez les populations de migrants.

Cette crise humanitaire pose de nombreuses questions à la Grèce :

a. La capacité de l'administration grecque à faire face au volume de cette vague migratoire et les limites d'une réponse adéquate se traduisent par un accès limité au chauffage, à la lumière et à l'électricité.

\footnotetext{
${ }^{15}$ Quatrième rapport sur la Grèce, ECRI, Conseil de l’Europe, 15 septembre 2009 [CRI(2009)31], §155.
} 
b. Les camps accueillent deux fois plus de personnes que normalement, et il y a une augmentation sensible du nombre de sans-abris.

c. Il y a une dégradation du climat sécuritaire. Avec $40 \%$ des jeunes en Grèce qui sont sans emploi, en plus d'un système de santé hautement fragilisé, les fonds nécessaires à la sécurisation du pays via la police ne sont tout simplement pas disponibles.

d. Le climat social se détériore au sein des camps entre différents groupes ethniques et entre les migrants et la population locale. Le parti d'extrême droite Aube Dorée entend organiser une résistance à l'implantation de camps. Un camp de réfugiés sur l'île de Chios a été attaqué au cocktail molotov en novembre 2016. Il y a un gros enjeu politique : le parti néonazi Aube Dorée avait fait $16 \%$ à Athènes lors des élections municipales de 2014. Il tire parti de ces crispations. On ne peut pas exclure le risque d'une explosion sociale et politique. Les conditions sont réunies pour une tempête provoquée par la crise financière, la crise des réfugiés et les tensions de politique intérieure.

e. La coordination et le financement du travail des ONG. C'est ici que l'action sociale de l'Église de Grèce s'est fait remarquer dans l'aide et le soutien apporté aux migrants et aux réfugiés, dans l'accès au logement, les vêtements et la nourriture. Il faut noter l'action de l'Eglise de Grèce au travers de son ONG Apostoli et les initiatives locales portées par des prêtres de paroisse, l'un des plus connus étant sans doute Papa Stratis. Ce dernier, mort en 2015, est rapidement devenu une figure emblématique de l'engagement ecclésial au profit des plus vulnérables prolongeant l'activité caritative qu'il avait lancée au début de la crise économique grecque et en l'appliquant aux réfugiés.

Au plus fort de la crise économique et migratoire, en 2016, alors que les attaques à l'encontre des migrants progressaient, la rencontre du pape François, du patriarche œcuménique Bartholomée et de l'archevêque Jérôme d'Athènes avait apporté un signe fort à la fois politique et religieux. S'étant retrouvés sur l'île 
de Lesbos, les trois prélats chrétiens s'étaient rendus au centre de rétention de Moria en allant à la rencontre des migrants. Il s'agissait, comme l'indique bien le texte de leur déclaration commune en date du 16 avril 2016, d'un appel collectif à dépasser les tentations du repli :

Ensemble, nous plaidons solennellement pour une fin de la guerre et de la violence au Moyen-Orient, pour une paix juste et durable et pour le retour honorable de ceux qui ont été contraints à abandonner leurs maisons. Nous demandons aux communautés religieuses d'accroître leurs efforts pour recevoir, pour assister et pour protéger les réfugiés de toutes les confessions; et que les services d'assistance religieux et civils travaillent à coordonner leurs initiatives. Car, tant que le besoin perdure, nous exhortons tous les pays à étendre l'asile temporaire, à offrir le statut de réfugié à ceux qui sont éligibles, à accroître leurs efforts d'assistance et à travailler avec tous les hommes et toutes les femmes de bonne volonté en vue d'une fin rapide des conflits en cours. ${ }^{16}$

La crise migratoire qui traverse aujourd'hui la Grèce permet une mise en lumière du caractère particulièrement complexe qui unit d'une part religion et identité nationale et $d^{\prime}$ autre part religion et engagement humanitaire ${ }^{17}$. Servant de prétexte à des discours politiques extrêmes, voire extrémistes, et à recristalliser la fusion religio-identitaire constitutive de l'identité nationale grecque à partir du XIXe siècle, il apparaît que les recherches scientifiques concernant le pluralisme religieux en Grèce s'intéressent davantage aux effets de la sécularisation issus du contexte européen que des mutations du paysage religieux à proprement parler (Dimitriadi et Sarantaki 2018). À cet égard, le think tank grec Eliamep et ses chercheurs, comme Effie Fokas qui dirige le programme «Grassrootsmobilise

\footnotetext{
16 «Déclaration commune de Sa Sainteté Bartholomée, Patriarche œcuménique de Constantinople, de Sa Béatitude Hyeronimos, Archevêque d'Athènes et de toute la Grèce et du Saint Père François ", Lesvos, 16 avril 2016. http://w2.vatican.va/content/francesco/fr/speeches/2016/april/documents/papafrancesco 20160416 lesvos-dichiarazione-congiunta.html

${ }^{17}$ De récents travaux, notamment du professeure Anna Triandafyllidou, suivent de manière très détaillées l'évolution des phénomènes migratoires en Grèce. Voir l'un de ses plus récents rapports publié avec E. Gemi : "Migration in Greece : Recent Developments in 2018 », Report prepared for the OECD Network of International Migration Experts, octobre 2018.
} 
project », montrent parfaitement que le débat autour du pluralisme religieux est avant tout le résultat d'un façonnage issu de la jurisprudence européenne, plutôt que de l'évolution de la diversité religieuse à proprement parler. En effet, la nature même de l'immigration récente en Grèce, le fait que la Grèce soit à la fois une porte d'entrée en Europe et de sortie vers d'autres pays européens - aux frontières plus ou moins perméables - et l'intégration de communautés d'immigrés légaux de plus en plus nombreuses dans le pays font suggérer que la réalité migratoire va faire ou fait déjà - évoluer la composition religieuse du pays. L'intégration des demandeurs d'asile reste quant à elle plus précaire. À long terme, ce n'est pas tant la crise migratoire contemporaine qui touche la Grèce, que le fait que la Grèce soit devenue un pays d'immigration de longue durée qui certainement va amplifier la question du traitement de son pluralisme religieux comme interrogation centrale dans les années à venir ${ }^{18}$. L'Etat grec, en poursuivant les trajectoires entre autres législatives et éducatives que nous avons soulignées, sera d'autant plus obligé à prendre en compte la nouvelle réalité religieuse du pays.

\section{Conclusion}

La question du pluralisme en Grèce a beaucoup évolué depuis le retour de la démocratie en 1974. De nombreux chantiers restent cependant à investir pour une meilleure prise en compte de la diversité culturelle et religieuse du pays. Selon Prodromos Yannas, ce processus ne pourra être renforcé que par une meilleure intégration régionale de la Grèce et par son alignement sur les standards démocratiques européens. Selon cet auteur :

Le processus a été rendu possible grâce à la Cour Européenne des Droits de l'Homme, ainsi que par les efforts des gouvernements grecs successifs dans la mise en place de mesures de discrimination positive et de politiques favorables aux intérêts spécifiques des minorités religieuses (Yannas 2010 : 123-124).

\footnotetext{
${ }^{18}$ Ibid., p.40.
} 
Néanmoins, il convient de reconnaître l'hégémonie religieuse et culturelle de l'orthodoxie, qui agit comme un régulateur essentiel de la place des communautés minoritaires dans le pays. Même si l'orthodoxie ne possédait pas une position dominante, en tant que religion d'État, elle n'en resterait pas moins un marqueur constitutif de l'identité nationale grecque. Dès lors, la reconnaissance d'un modèle sociétal pluraliste en Grèce dépend de la capacité des minorités culturelles et religieuses du pays à ne pas entrer en compétition avec les définitions identitaires dominantes. Dans le contexte de crise économique, il semble nécessaire de renforcer cet axe, afin que le développement de discours ultranationalistes et racistes ne viennent effacer les progrès dans la lutte contre les discriminations.

De même, la normalisation des relations de la Grèce avec ses pays voisins aura pour effet d'agir sur l'évolution des représentations des composantes de cette diversité. L'objectif de cette intervention consistait à mettre en lumière les effets contradictoires de la crise migratoire sur le pluralisme en Grèce. Le paradoxe essentiel réside dans la tension entre le phénomène de fixation identitaire au travers du religieux et la mission proprement caritative de la religion. La crise migratoire a largement servi à l'exacerbation des tendances identitaires et exclusivistes de la religion et notamment de l'orthodoxie. Georges Prévélakis abonde en ce sens : «L'Église autocéphale grecque ne peut échapper totalement à l'influence de l'État grec; elle se trouve tiraillée entre sa volonté de préserver ses traditions et les pressions de l'idéologie nationaliste » (Prévélakis 2017 : 164). 


\section{Bibliographie}

Akgönül, S. (1999). Une minorité, deux États: la minorité turco-musulmane de Thrace occidentale. Istanbul, Isis.

Anagnostou, D. et A. Triandafyllidou. (2006). Regions, Minorities, European Integration: A Case of Study on Muslim in Western Thrace, Greece. ELIAMEP, Project Report.

Banchoff, T. (dir.) (2008). Religious Pluralism, Globalization, and World Politics. New York, Oxford University Press.

Berger, P. et T. Luckmann. (1967). "Aspects sociologiques du pluralisme». Archives de sociologie des religions, $\mathrm{n}^{\circ} 23, \mathrm{p}$. 117-127.

Bertrand, G. (2003). Le Conflit Helléno-Turc. La Confrontation de deux Nationalismes à l'aube du XXIème siècle. Paris, Maisonneuve \& Larose.

Bruneau, M. (dir.) (1998). Les Grecs pontiques, diaspora, identité, territoires. Paris, CNRS Éditions.

Clogg, R. (dir.) (2002). Minorities in Greece. Aspects of a Plural Society. Londres, Hurst and Co.

Dimitriadi, A. et A.-M. Sarantaki. (2018). "The refugee 'crisis' in Greece : politicisation and polarisation amidst multiple crisis ». CEASEVAL Research of the Common European Asylum System, n¹1, novembre 2018.

Kazarian, N. (2012). Chypre, Géopolitique et Minorités. Paris, L'Harmattan.

Kazarian, N. (2018). L'orthodoxie. Paris, Eyrolles.

Meinardus, R. (2002). «Muslims: Turks, Pomaks and Gypsies ». Dans R. Clogg (dir.), Minorities in Greece. Aspect of a Plural Society. Londres, Hurst and Co., p. 81-93.

Prévélakis, G. (2017). Qui sont les Grecs? Une identité en crise. Paris, CNRS Éditions. 
Roudometof, V. (2010). «The Evolution of Greek Orthodoxy in the Context of World Historical Globalization ». Dans V. Roudometof et V. N. Makrides (dir.), Orthodox Christianity in 21 ${ }^{\text {st }}$ Century Greece. Farnham, Ashgate, p. 2138.

Tsaliki, E. (2017). "Religious Education in Greece Under the Scope of Interculturalism ». Journal of Education and Training, vol. 4, n 1, p. 40-54.

Ursinus, M. O. H. (1993). « Millet». Encyclopédie de l'Islam.t. 7. Leyde, Brill.

Ware, K. (2002). "Old Calendarists ». Dans R. Clogg (dir.), Minorities in Greece. Aspect of a Plural Society. Londres, Hurst and Co, p. 1-23.

Yannas, P. (2010). «Non-Orthodox Minorities in Contemporary Greece: Legal Status and Concomitant Debates between Church, State and the International Community ». Dans V. Roudometof et V. M Makrides, Orthodox Christianity in 21 ${ }^{\text {st }}$ Century Greece. Farnham, Ashgate, p. 111-128. 